\title{
Hippocampal Representational Organization and Spatial Context
}

\author{
S.J.Y. Mizumori, ${ }^{*}$ K.E. Ragozzino, B.G. Cooper, \\ and S. Leutgeb
}

Department of Psychology, University of Utah, Salt Lake City, Utah

\begin{abstract}
The hippocampus appears to undergo continual representational reorganization as animals navigate their environments. This reorganization is postulated to be reflected spatially in terms of changes in the ensemble of place cells activated, as well as changes in place field specificity and reliability for cells recorded in both hilar/CA3 and CA1 regions. The specific contribution of the hilar/CA3 region is suggested to be to compare the expected spatial context with that currently being experienced, then relay discrepancies to CA1. The properties of CA1 place fields in part reflect the spatial comparisons made in the hilar/CA3 area. In addition, CA1 organizes the input received from the hilar/CA3 place cells according to different temporal algorithms that are unique to different tasks. In this way, hippocampus helps to distinguish temporally one spatial context from another, thereby contributing to episodic memories. Hippocampus 1999;9:444-451. ㅇ 1999 Wiley-Liss, Inc.
\end{abstract}

KEY WO RDS: hippocampus; reorganization; place fields

\section{INTRODUCTION}

Spatial knowledge is an integral aspect of most, if not all, of our behaviors. Such knowledge is to varying degrees anchored to, or a reflection of, our external world. Studies on the dynamic aspects of representations in sensory systems (summarized by M erzenich and deCharms, 1996) show that changes in external sensory input result in continuous changes in representations across multiple levels of sensory processing. Furthermore, the finding that the tuning of sensory neurons in cortex can change dramatically and systematically depending on internally-generated constructs such as attention or expectation, suggests that the past history of neural activity importantly influences our perceptions. Such widespread and diverse effects are thought to reflect the fact that perceptual processing is a dynamic and interactive exchange amongst many structures within a larger neural system.

A logical extension of this sensory neural systems analysis is that operation of a navigational system during spatial learning, which likely depends on the continual analysis of dynamic (internal and external) sensory environments, also involves a well-orchestrated effort by multiple

Grant sponsor: NSF; Grant numbers: BNS 9120784, DG E9616182; Grant sponsor: NIH ; Grant numbers: MH58755, MH11998.

*Correspondence to: Dr. Sheri J. Y. Mizumori, Department of Psychology, 502 Social and Behavioral Science Bldg., U niversity of U tah, Salt Lake City, UT 84112. E-mail: mizumori@behsci.utah.edu Accepted for publication 3 May 1999 levels of representation in the brain. Specifically, given the nature and complexity of the integrative functions that mediate spatial learning, it would be expected that successful navigation depends upon the cooperation of multiple cortical and subcortical systems. These systems could be similarly affected by changes in external sensory input, and systematically distorted during attentional shifts. By processing within such a broad neural system, goal-directed navigation can be highly adaptive since current spatial context information can be readily interpreted to affect learning, planning, and acting.

We have tested the broad neural systems view of adaptive navigation by studying across multiple brain areas neurophysiological correlates of navigation in unrestrained rats as they perform a spatial learning task. Extensive converging evidence (from other investigators as well as ourselves) shows that at least the hippocampus is clearly involved in spatial learning. Electrophysiologically, the pyramidal cells of hippocampus display location and direction selective firing (referred to as place fields) when animals engage in specific goal-directed behaviors (e.g., O'Keefe, 1976; M CNaughton et al., 1983; M izumori et al., 1989). M any would now agree that hippocampal place fields dynamically reflect the integration of organism- and environment-centered information (e.g., Feigenbaum and Rolls, 1991; 0 'M ara et al., 1994; Knierim et al., 1995; M cN aughton et al., 1996; Wiener et al., 1995; Gothard et al., 1996). A more debated issue concernsthe significance and nature of this integration.

To address the issue of the functional significance of place cells to spatial learning, we have compared hippocampal place and directional codes with place and directional codes of striatal (Lavoie and Mizumori, 1994; M izumori et al., 1996, 1998), amygdalar (Pratt and M izumori, 1998), collicular (Cooper et al., 1998), and thalamic neurons (M izumori and W illiams, 1993). In doing so, similarities in spatial codes have been revealed: for example, place fields from diverse brain structures are sensitive to changes in the visual spatial environment and show directional biases on the radial maze. There are also clear differences in place fields across structures in terms of the specificity of the location code, and how different place fields respond to 
environmental change (described below). These differences suggest that while multiple structures represent spatial context, they do so for different computational reasons. For instance, the hippocampus might help organisms to learn about new spatial contexts (N adel et al., 1985), while place codes in other structures, for example, the striatum, may be used to guide the process of monitoring an animal's ongoing behavior (M izumori et al., unpublished data). With respect to hippocampus, we extend the spatial context interpretation of place fields by arguing that the reason hippocampus represents spatial context is to allow animals to learn about important changes in the external spatial environment, as well as to learn about changes in the meaning of, or changes in attention to, constant environmental cues. The hippocampus accomplishes this by comparing the expected spatial context with the one currently being experienced, then transmitting a message to the cortex, which indicates the nature and extent of the discrepancy (but see also G othard et al., 1996). "Expected" spatial context in our case is meant to refer to an information packet that reflects past knowledge of the spatial relationship of salient cues (visual and nonvisual) in a familiar environment, knowledge of the appropriate strategy for solving the task, and information concerning the past motivational consequences of spatially-defined areas of an environment. It is worth noting that consideration of context information has been shown to lead to the development of simulated place fields (Wallenstein and $\mathrm{H}$ assel mo, 1997). O ur hypothesis makes a number of predictions regarding the behavior of hippocampal place cells, individually or as a population. Some of these are supported by extant data, and others remain to be explored.

\section{USE OF FAMILIAR SENSORY INFORMATION IN FLEXIBLE WAYS}

A diversity of hippocampal place cell responses to changes in spatial context has been described by many researchers (e.g., Muller and Kubie, 1987; Knierim et al., 1995; M arkus et al., 1995; Sharp, 1997). In our hands, some $40-60 \%$ of hippocampal place cells retain essentially the same place fields in rats performing at asymptote when, following initial exposure to a familiar room, access to visual cues is denied. These neurons might encode memory-driven (expected) features of spatial context. In contrast, other cells change almost immediately in response to alterations in the spatial environment, and might identify for hippocampus the current context. Cells that maintain the same place fields for a while, but eventually change after many minutes of exposure to the dark environment, might reflect hippocampal integration of both expected and current context. Such response diversity is not likely to be a reflection of expected variability when recording populations of place cells, but rather it may reflect the relatively unique type of functional integration that hippocampus performs. To examine this idea, we have compared the responses of hippocampal place cells with responses of place cells found in other brain areas. After recording from over 50 caudate-putamen place cells, we find that all of those tested thus far change almost immediately following the onset of darkness (Fig. 1). Similarly, location-selective neurons in the superior colliculus show rather immediate responses to unexpected darkness in familiar environments (Cooper et al., 1998). Therefore, not all place codes show the same response to visual manipulations as we have observed in hippocampus.

The range of stability of place representation in hippocampus during major changes in a familiar visual environment (i.e., darkness) may be a prerequisite for flexible processing of spatial context information. Based on prior learning, representations of the expected spatial environment may form a stable contextdefined framework (or reference frame) within which current sensory information (real or perceived) is processed. In other words, hippocampus can flexibly consider multiple sensory configurations with respect to a spatial reference frame that is based on information concerning the expected spatial context. In this way, animals may experience perceptual constancy across environments, which in turn could account for the ability to generalize across spatial situations.

The neural expression of expected and current context may be manifested as particular patterns of activity across large neocortical areas. This activity reflects associations between memory representations and current multisensory data, as well as between memory representations and heading direction or self-motion information derived from neocortical and thalamic structures (e.g., M izumori and Williams, 1993; Chen et al., 1994; Taube, 1995). The neocortical information defining expected and current sensory contexts likely arrives in hippocampus proper via entorhinal afferents. Evidence suggests that place cells in both CA1 and the hilar/C 33 area receive entorhinal afference. Feedback concerning the current internal motivational and movement state could enter into hippocampus via subcortical afferents (from hypothalamic and mammillary structures) to the dentate gyrus/CA3 region (e.g., Vertes, 1992; Vertes et al., 1995). W hen animals are performing at asymptote on a familiar task, the hippocampal signal may serve to ultimately strengthen synapses of the currently active cortical networks that define the expected spatial context. This is analogous to the view that hippocampus may serve to "consolidate" long-term memory stores in cortex (e.g., Squire et al., 1984). H owever, if a sufficient difference between expected and current spatial context is detected, the resultant hippocampal signal destabilizes the neocortical network activity that represented the most recent expected context. This may allow a closely related, but different, spatial context definition to emerge into a stable state where it can guide future comparisons. In contrast to the situation that exists during asymptote performance, a memory representation of the expected spatial context may not be immediately available for use by hippocampus during the initial stages of new spatial learning. This should result in proportionately greater representation of current context during new learning. Thus, a greater proportion of hippocampal place fields should 
change in response to context change manipulations rather than remaining stable.

INTRAHIPPOCAMPAL

SPECIALIZATIONS DURING SPATIAL LEARNING

A system that continually analyzes the difference between expected and current spatial context probably does so in stages. The initial computation to be performed might be to sort or orthogonalize the afferent information (perhaps in the dentate gyrus/CA3 area) via algorithms such as pattern separation ( 0 'Reilly and McClelland, 1994; Rolls, 1996), which, based on past experience, selects the relevant sensory/perceptual inputs for comparison. Then, by virtue of its intrinsic neural architecture, the hilar/CA3 area makes a unique contribution to the comparison of expected and experienced contexts by making the determination as to whether or not a difference exists. Such current and expected information is dynamically changing during active navigation. As a result, a mechanism is required whereby information can be held briefly in an "active" stateso that comparisons can be made. Such an active state would need only persist for a short period of time relative to that required of working memory buffers since, during a single trial of a navigation task, there should be a continual series of spatial context comparisons that need to be made. The recurrent excitatory loops in CA3 may serve this purpose (Swanson et al., 1980, 1981).

The results of the analysis performed in CA3 need to be temporally organized to allow for meaningful patterned output to efferent targets (e.g., prefrontal cortex). Temporal processing in the hippocampus has been suggested by different investigators (e.g., Olton, et al., 1979; Rawlins, 1985; Kesner, 1991). A selective role for CA1 in the temporal organization of hippocampal efferent messages has recently been suggested (e.g., W iener and Korshunov, 1995) since CAl units discharged in response to task-specific behaviors, and this firing was not related to the location of the animal. These spatial and nonspatial correlates have been observed for the same cell tested in different situations (e.g., Wiener et al., 1989), and may reflect the temporal sequence of behaviors exhibited (e.g. H ampson et al., 1993). The apparent relationship of CA1 discharge to the temporal sequence of behavior may occur because CA1 (like CA3) receives memorybased contextual information concerning the appropriate strate gies required to solve the task. This information is important to CA1 since the current cognitive strategy dictates how spatial output from CA3 is to be organized for distribution to other brain areas. Indeed, Wilson and Tonegawa (1997) found that the temporal coherence of discharge by CA 1 place cells with overlapping place fields increased after learning. O ne could build on this initial finding by predicting that there are additional levels of complexity to the temporal coherence code. For example, the relative magnitude of population responses associated with different locations could vary in nonrandom ways with the extent of temporal coherence. It is also possible that CA1 integrates spatial information over more extended periods of time, thereby defining episodes according to specific contexts. Such a process may not require a specific neural architecture such as recurrent excitatory loops. Rather different contexts may invoke different states of activation of CAl cells, which are maintained until the real or perceived context changes. CAl output that is associated with a given period of activation may help neocortex to incorporate specific episodes or events into long-term store. In this way, hippocampus might contribute to the spacetime integration necessary for episodic memories.

Is temporal coherence, or temporal organization, of place field information unique to CA1? To our knowledge, a systematic evaluation of other populations of place cells has not yet been performed. Furthermore, it would be of interest to determine whether cells that become temporally coherent have place fields that persiste after context changes in familiar environments, place fields that changed immediately in different contexts, or place fields that are intermediate in stability. We predict that coherence should be greatest for place cells that integrate expected and current context, i.e., those cells that change gradually after context change.

The specific functional distinction proposed for CA1 and hilar/CA3 place cells is supported by many of our earlier reports. Place cells recorded in the hilar/CA3 region tended to show more selective place fields than those recorded in CA1 (Barnes et al., 1990; Mizumori et al., 1989, 1996; Mizumori and Cooper, 1995). H owever, the magnitude of the difference can vary as a function of variations in task procedure (M izumori et al., 1996). Consequently, statistical confirmation of this difference may not

FIGURE 1. A comparison of the effects of darkness on place cells recorded in CA1, hilus/CA3, and the striatum. Each panel graphically represents the locations visited by the animal (dots) as well as the relative local firing rates of each cell (circles) recorded as animals perform trials 1- 5 under standard light conditions, trials 6- 10 under complete darkness, and trials 11-15 under standard light conditions. Each plot contains superimposed data from five trials. Vectors indicate the direction of diode (presumably animal) movement on the maze. (TOP) This CA1 place field did not noticeably change specificity across the three test conditions, although the mean firing rate may have been reduced slightly when the rat ran in darkness. In contrast, the two hilar/CA3 cells ( 2 middle rows) and the striatal place cell (bottom) showed dramatic and clear reorganization in darkness. The first hilar/CA3 cell coincidentally exhibited a place field in precisely the same location as that shown above for the CA1 place cell (recorded from a different rat). H owever, in contrast to the response of the CA1 cell, this hilar/CA3 place cell essentially stopped firing during dark trials. The original place field can be seen to return during subsequent control light trials. The second hilar/CA3 place cell continued to fire during dark trials, although the spatial distribution of firing changed dramatically. Interestingly, while this cell retained the same movement correlate (i.e., it fired only during turns at the arm ends), the turn correlated was no longer restricted to a single maze arm. It was as if the cell continued to receive normal idiothetic information, but lost its "anchor" to the spatial environment. During the last five baseline trials, it can be seen that the original correlate had returned. This pattern suggests the hilar/CA3 region and the striatum place cells encode information relevant to the current spatial context (although for different reasons; see text), while CA1 may be driven more by the mnemonic aspects of context analysis. 


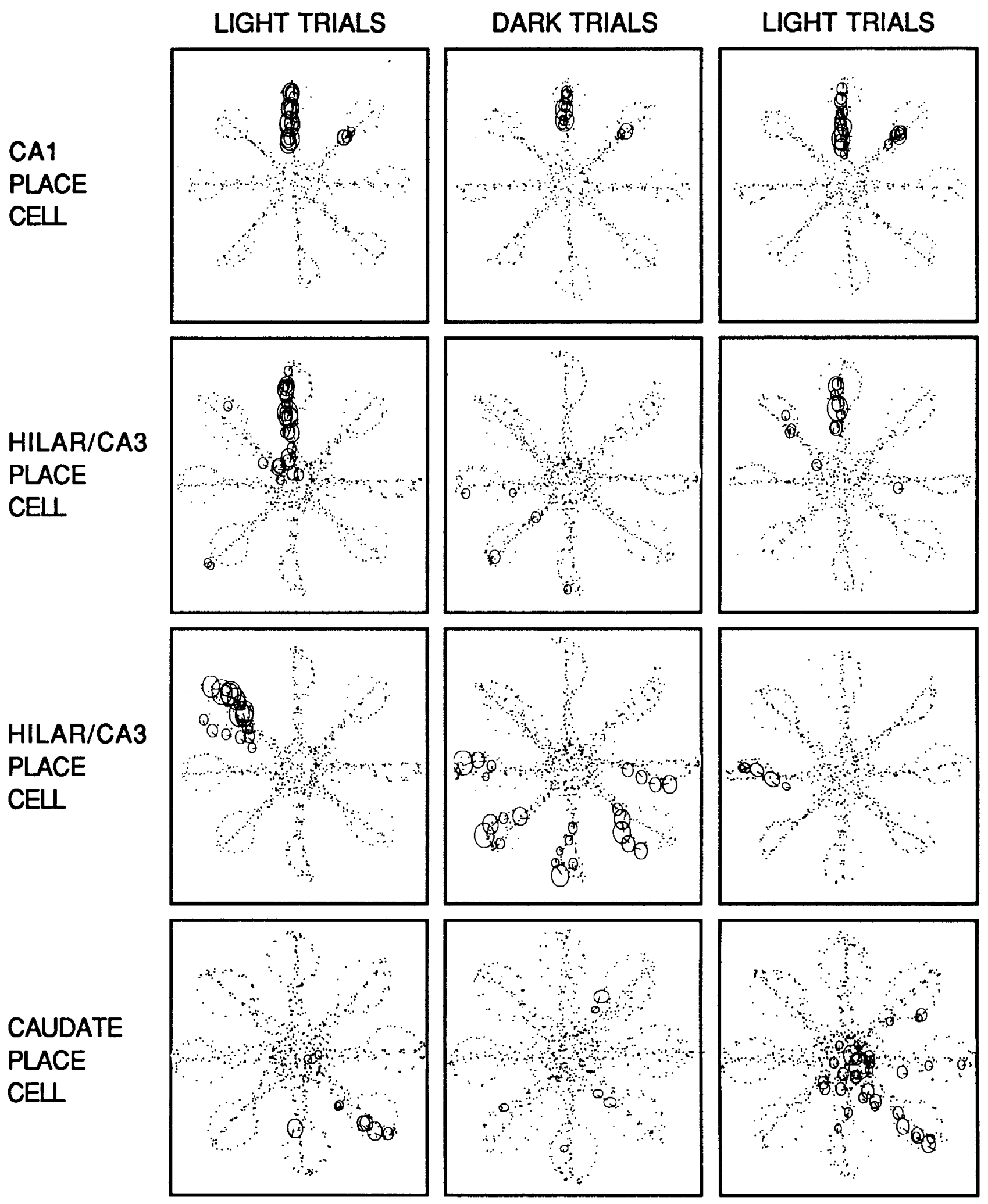

Figure 1.

always be obtained (e.g., M izumori and Kalyani, 1997). In our study, statistical reliability was not achieved when cells were recorded during the acquisition of a spatial working memory task, but was obtained when animals were performing at asymptote. In the latter condition, the distinction between $\mathrm{CA} 1$ and hilar/CA3 place fields was mainly due to the fact that the hilar/CA3 cells increased specificity; as a population, CA1 place fields maintained the same degree of specificity before and after spatial learning 
(M izumori et al., 1996), although the reliability of the CA1 fields increased during the spatial learning phase (Mizumori and Kalyani, 1997).

The results of the comparison between $\mathrm{CA} 1$ and hilar/CA3 place fields are consistent with the proposed view of hippocampus as a spatial context comparator. O ne could imagine that during initial spatial learning, the difference between the expected and currently experienced spatial context/perception is greatest. As learning progresses, the difference between expected and current spatial context/perception decreases, resulting in more precise spatial codes. Consequently, hilar/C A3 cell location-specific discharge would be expected to vary with errors as spatial learning takes place. If the development of highly selective, reliable hilar/CA3 place fields indeed reflects a good match between expected and current environments, then one would predict that significant disruption of the familiar spatial context would result in a destabilization of the hilar/CA3 code. Data are shown in Figure 1 that support this prediction. Prolonged exposure to the new environment should lead to subsequent re-stabilization of the hilar/CA3 place codes.

In contrast to hilar/CA3 place fields, CA1 place field properties may or may not necessarily be expected to correlate with errors, depending on the nature of the new task demands. That is, the temporal structure of CA1 output messages may be established independent of the degree of match relayed by the hilar/CA3 region, and this temporal organization is expected to be established early on in training. (Presumably animals learn to adopt a cognitive algorithm before they learn and remember the details of which spatial relationships are relevant.) Therefore, temporal organization is predicted to change when a new cognitive structure is imposed (e.g., when animals must use familiar cues in new ways, or when animals enter an entirely new learning task), but not necessarily when animals use the same cognitive structure across multiple environments (e.g., when animals perform the same memory task in different rooms).

Individual CA1 place fields should be expected to be more variable in their spatial coding than the message conveyed in the temporal organization of $\mathrm{CA} 1$ output since the computational signal of a given neuron may change depending on attention. $M$ aybe, it is for this reason that one observes a correlation between errors and place field reliability more readily between errors and place field specificity (e.g., M arkus et al., 1995; M izumori et al., 1996) during acquisition of a spatial task. This correlation may reflect the gradually increasing reliability of CA3 input with learning, which in turn reflects an increasingly well-defined context interpretation. This is not to say that CAl place field specificity measures would not be sensitive to changes in spatial context. There are, in fact, many documentations of contextdependent changes in the size of CA1 place fields when monitored over relatively short periods of time (e.g., M uller and Kubie, 1987; Wilson and McN aughton, 1993; M arkus et al., 1995; O 'Keefe and Burgess, 1996; H etherington and Shapiro, 1997). H ow these changes relate to behavioral errors during an explicit learning task over days to weeks is less certain (M izumori and Kalyani, 1997).

That CA1 and hilar/CA3 regions have different relationships to the number of errors is also observed following medial septal inactivation (M izumori et al., 1989). Temporary deafferentation produces significant impairment of choice accuracy on a spatial working memory task at a time when the specificity of hilar/C A3, but not CA1, place fields are disrupted. It should be noted that although CA1 place field specificity was not disrupted with this procedure, the temporal aspects of the output message (e.g., temporal coherence) by CA1 may have been abnormal, contributing to impaired performance.

REPRESENTATIONAL REORGANIZATION IS THE RULE RATHER THAN THE EXCEPTION

As animals continually encounter different environments and situations, the relative novelty of sensory cues, and the extent to which the current context matches familiar ones, will vary dynamically. This change in relative influence may reflect a continual shift in attention across different behavioral situations. W ith each situation, one could imagine that a different subset of afferents are activated, and the selection of the subset may be influenced by experience. This line of reasoning predicts that as animals experience different settings, representations will reorganize accordingly. Reorganization may be manifested at different levels from changes in the properties of individual place fields to changes in the relative specificity and reliability of the spatial code across distinct populations of place cells. Another form of reorganization involves not so much changes in spatial representations, but rather changes in temporal organization of spatial data, as shown by W ilson and Tonegawa (1997).

C onsider a specific situation where an animal is placed in a new environment and task in which the appropriate cognitive strategy remains to be determined (i.e., new learning). As described above, one would expect both CA1 and hilar/CA3 cells to change from their prior state upon entering the new situation. Place fields in CA1 indeed tend to reorganize, or "remap," during the initial minutes of exposure to a new environment, with comparatively minor modifications after that (e.g., Muller and Kubie, 1987; Wilson and M cN aughton, 1993; M arkus et al., 1995; O 'Keefe and Burgess, 1996; Hetherington and Shapiro, 1997). We interpret this to mean that CA1 has established an initial algorithm for organizing hippocampal output. We predict that hilar/CA3 place cells would show similarly rapid reorganization if animals are simply placed in a different environment. If, however, specific spatial relations need to be learned, then our prediction is that hilar/C A3 place cells would show more substantial changes in spatial correlates across longer periods of time. The length of time required reflects the time needed to identify and learn which spatial relationships are most salient.

A second scenario occurs when animals learn to use familiar environmental information according to new task rules. In this situation, onewould expect to observe representational reorganization in both the hilus/CA3 region and in the $\mathrm{CA} 1$ area. The new rules dictate a new definition of the spatial context, which in turn 
determines a new relevant algorithm for temporal organization. We have some initial evidence that place fields of hilar/CA3 place cells increase specificity when a task is switched from forced choice to spatial learning while animals perform in a familiar environment (M izumori et al., 1996). In that same study, overall specificity the entire population of CA1 place cells recorded did not change when the task changed. H owever, since different cells were recorded during the forced choice and spatial memory tests, there may have been a sampling bias. Also, the new organization of spatial information that was required during spatial learning should have resulted in a change in temporal organization of CA1 output rather than a change in place field specificity. As indicated above, the correlation between CA1 place field reliability and errors may reflect more reliable hilar/CA3 input. It remains an open issue whether $C A 1$ reorganizes in this situation.

A third type of environmental encounter is one in which an animal detects a change in a once familiar environment, but nevertheless perceives the expected task demands to be fundamentally unchanged. Experimentally, this situation has been investigated when familiar cues have been scrambled or removed, or when darkness in suddenly imposed as animals are moving about a familiar environment. Animals detect the change in cues but (at least initially) still try to solve the task according to learned rules. O ur view of the functional distinction between CA1 and the hilar/CA3 regions predict that hilar/CA3 place field activity should be more readily affected by changes in either the external spatial environment or internal state than CA1 place fields. Dissociation of $\mathrm{CA} 1$ and hilar/CA3 responses following a change in signals relaying internal state information (presumably via septal afferents) has already been described (M izumori et al., 1989). To address a similar prediction following changes in the external environment, we recently compared CA1 and CA3/hilar place cell responses to imposed darkness after animals had learned a standard spatial working memory task on an 8-arm radial maze. These data were obtained from control animals for studies to be described in other reports. The specific analyses described below, however, are unique from what will be presented elsewhere.

The animals were mature, adult male rats (Fischer-344, W istar, Long-Evans, or Fischer-Brown N orway hybrids) of 9-16 months of age. All were trained to retrieve chocolate milk reward located on the ends of each maze arm. The correct strategy for solving the task was to select each arm once per trial; reentries were counted as errors. Animals were trained to perform 8-15 trials per day, then multiple stereotrode recording electrodes were surgically implanted. After recovery from surgery, animals performed 15 trails daily. The effects of changes in spatial context on place fields were tested by allowing rats to first perform 5 trials under standard maze conditions. The lights illuminating the maze were turned off, and rats then performed trials 6-10 in complete darkness. Recovery trials 11- 15 were performed with the room lights on.

During baseline (asymptote) performance (i.e., trials 1-5), hilar/CA3 place fields ( $n=61$ neurons) showed greater information content scores (Skaggs et al., 1993) than CAl ( $n=183$ neurons), $F(1,242)=10.28, P<.01$. Since this score is thought to reflect the degree of location selective discharge, this result is consistent with our earlier findings of greater location specific codes by hilar/CA3 place cells than CA1 place cells. In addition, an analysis of the spatial covariance (i.e., reliability) of the fields across trials within a recording session revealed significantly greater covariance for hilar/CA3 place cells than CA1 place cells, F $(1,242)=4.47, P<.05$. There were no significant differences between areas in terms of the proportion of cells that were classified as place cells, the mean firing rate, or the extent to which these two populations of place fields were directional.

The extent and type of place field changes observed during dark trials were quantified first by determining the proportion of place cells in each population that changed in darkness. Figure 2 shows that significantly more hilar/CA3 place cells changed during dark trials when compared to CA1 place cells, $\mathrm{X}^{2}=88.2, \mathrm{P}<.001$. The pattern of change was also different for the two populations of place cells. About $65 \%$ of the hilar/CA3 place fields that changed did so by showing no place fields in darkness, or by demonstrating a place field in darkness that was not detectable when the lights were on. In contrast, only $25 \%$ of CA1 place cells responded in one of these ways. CA1 place cells tended to become more or less location-specific, or they exhibited changed location preferences. The different patterns of responses suggest that, as a population, the hilar/CA3 region reorganizes its representational infrastructure in more dramatic ways than CAl during dark testing. Consistent with this interpretation are results of a comparison of the rotational deviation of the location of the $\mathrm{CA} 1$ or hilar/CA3 place fields in darkness relative to the field observed under the light condition (Fig. 2, bottom). O nly those cells that showed place fields in both light and dark conditions were compared. H ilar/CA3 place fields deviated morefrom the baseline comparison condition, $\mathrm{X}^{2}=7.96, \mathrm{P}<.01$. In summary, there seems to be converging evidence that hilar/CA3 place cells are more sensitive to context changes. This may reflect the fact that, relative to CA1, current context information is more critical for its neurocomputations.

\section{CONCLUDING REMARKS}

The hippocampus likely undergoes continuous representational reorganization as animals navigate and learn about their spatial environment. Factors that contribute to the reorganization could be changes in current motivational and movement states (mediated primarily via subcortical afferents) or changes in the expected and/or current spatial context (mediated via corticohippocampal afferents). W hile it is often assumed that changes in the pattern of excitatory afferents mediate the type and extent of reorganization, it is just as likely that experience-dependent effects on the inhibitory control of place field expression determine the plasticity observed at the representational level (Samsonovich and M cN aughton, 1997; also see relevant discussions in Wallenstein et al., 1998; Wallenstein and $\mathrm{H}$ asselmo, 1997; and Paulsen and M oser, 1998). It is argued here that it is only by understanding the controlling influences of internal cognitive states (e.g., attention, emotion, motivation, and drive) on hippocampal representational 

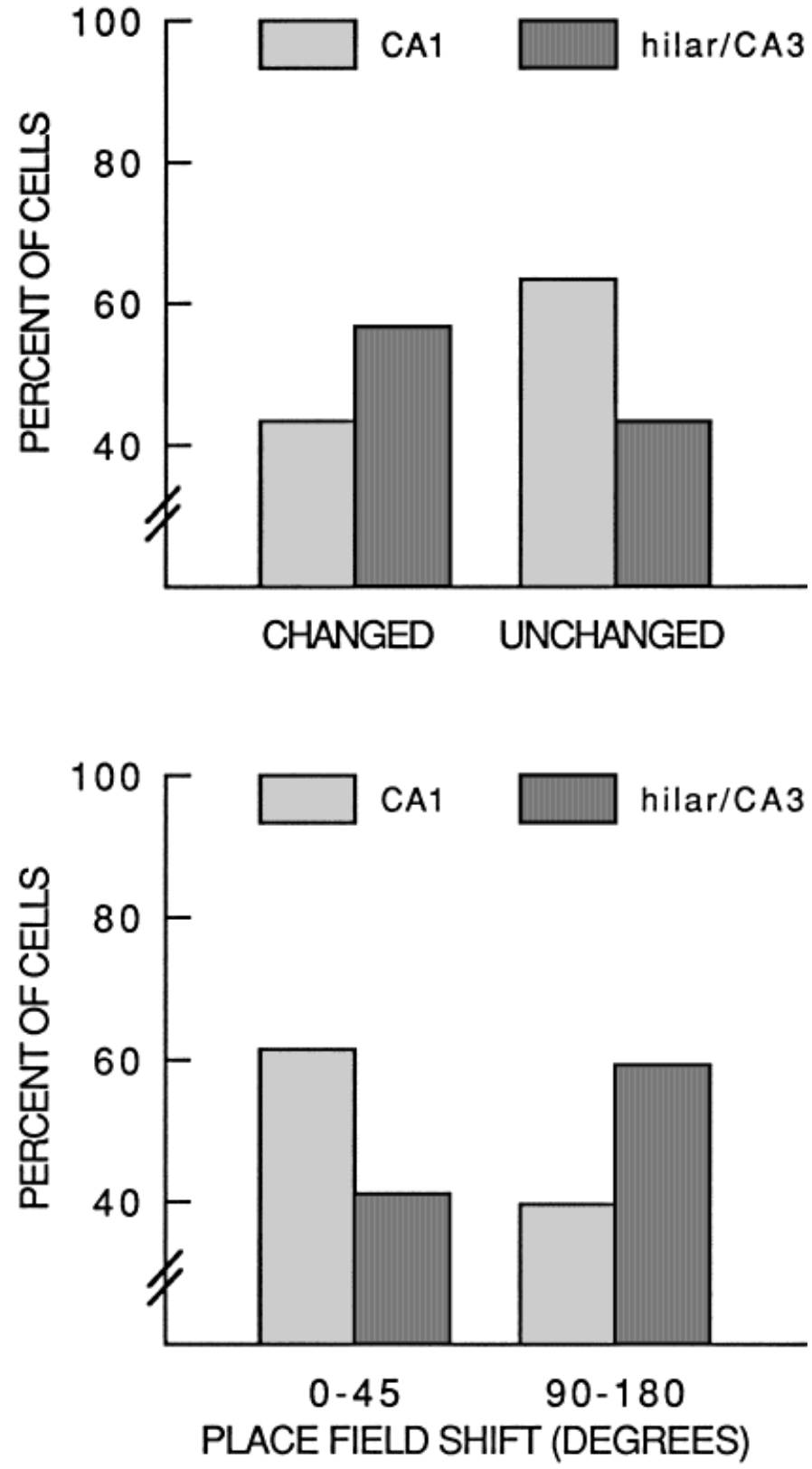

FIGURE 2. Top: The relative proportions of cells that were considered place cells from CA1 and the hilar/CA3 areas were about the same. It can be seen that a greater proportion of hilar/C 33 cells changed when compared to CA1 place cells. Bottom: Of the place cells that changed, the proportion of those that changed slightly (left) or dramatically (right). H ilar/CA3 place fields tended to shift more dramatically than CA1 place fields. Together, these data support the view that the hilar/CA3 reflects current behavioral accuracy than CA1.

plasticity that we can fully understand how experience affects spatial perception, learning, and performance.

\section{Acknowledgments}

We thank James Canfield and Wayne Pratt for comments regarding this manuscript.

\section{REFERENCES}

Barnes CA, M CN aughton BL, M izumori SJY, Leonard BW, Lin LH. 1990. C omparison of spatial and temporal characteristics of neuronal activity in sequential stages of hippocampal processing. Prog Brain Res 83:287-300.

Chen LT, Lin L-H, Green, EJ, Barnes CA, M CNaughton BL. 1994. $\mathrm{H}$ ead-direction cells in the rat posterior cortex I. anatomical distribution and behavioral modulation. Exp Brain Res 101:8-23.

Cooper BG, M iya DY, M izumori SJY. 1998. Superior colliculus and active navigation: Role of visual and non-visual cues in controlling cellular representations of space. H ippocampus 8:340-372.

Feigenbaum JD, Rolls ET. 1991. Allocentric and egocentric spatial information processing in the hippocampal formation of the behaving primate. Psychobiology 19:21-40.

Gothard KM, Skaggs WE, M CN aughton BL. 1996. Dynamics of mis-match correction in the hippocampal ensemble code for space: interactions between path integration and environmental cues. J N eurosci 16:8027-8040.

H ampson RE, H eyser CJ, D eadwyler SA. 1993. H ippocampal cell firing correlates of delayed-match-to-sample performance in the rat. Behav N eurosci 107:715-739.

Hetherington PA, Shapiro M L. 1997. H ippocampal place fields are altered by the removal of single visual cues in a distance-dependent manner. Behav N eurosci 11:20-34.

Kesner RP. 1991. N eurobiological views of memory. In: M artinez J J L, Kesner RP, eds. Learning and M emory: A Biological View. California: Academic Press, 499-547.

Knierim JJ, Kudrimoti HS, M CN aughton BL. 1995. Place cells, head direction cells, and the learning of landmark stability. J Neurosci 15:1648-1659.

Lavoie AM, M izumori SJY. 1994. Spatial-, movement, and rewardsensitive discharge by medial ventral striatum neurons of rats. Brain Res 638:157-168.

M arkus ET, Q in YL, Leonard B, Skaggs WE, M cN aughton BL, Barnes CA. 1995. Interactions between location and task affect the spatial and directional firing of hippocampal neurons. J N eurosci 15:70797094.

M cN aughton BL, Barnes CA, O 'Keefe J. 1983. The contributions of position, direction and velocity to singleunit activity in the hippocampus of freely-moving rat. Exp Brain Res 52:41-49.

M cNaughton BL, Barnes CA, Gerrard JL, Gothard K, Jung M W, Knierim JJ, Kudrimoti H, Q in Y, Skaggs WE, Suster $M$, W eaver KL. 1996. Deciphering the hippocampal polyglot: the hippocampus as a path integration system. J Exp Biol 199:173-185.

M erzenich M M , deCharmsRC. 1996. N eural representation, experience, and change. In: Llinas R, Churchland PS, eds. The M ind-Brain Continuum: Sensory Processes. Cambridge: M IT Press, 61-81.

M izumori SJY, Cooper BG. 1995. Spatial representations of dorsal caudate neurons of freely-behaing rats. Soc N eurosci Abst 21:1929.

M izumori SJY, Kalyani A. 1997. Age and experience-dependent reorganization during spatial learning. N eurobiol Aging 18:651-659.

M izumori SJY, Williams JD. 1993. Directionally-selective mnemonic properties of neurons in the lateral dorsal nucleus of the thalamus of rats. J N eurosci 13:4015-4028.

M izumori SJY, M cN aughton BL, Barnes CA, Fox KB. 1989. Preserved spatial coding in hippocampal CA1 pyramidal cells during reversible suppression of CA3 output: Evidence for pattern completion in hippocampus. J Neurosci 9:3915-3928.

M izumori SJY, Lavoie AM, Kalyani A. 1996. Redistribution of spatial representation in the hippocampus of aged rats performing a spatial memory task. Behav N eurosci 110:1006-1016.

M izumori SJY, Ragozzino KE, Leutgeb S, Cooper BG . 1998. Complementary roles of caudate and hippocampus during navigation: evidence from parallel single unit recordings. Soc N eurosci Abst 24:1911. 
M uller RU, KubieJL. 1987. T he effects of changes in the environment on the spatial firing of hippocampal complex-spike cells. J Neurosci 7:1951-1968.

N adel L, W illner J, Kurz EM . 1985. Cognitive maps and environmental context. In: Balsam P, Tomie A, eds. C ontext and Learning. H illsdale $\mathrm{N}$ ]: Erlbaum, 385-406.

0 'K eefe J. 1976. Place units in the hippocampus of the freely-moving rat. Exp N eurol 51:78-109.

0 'Keefe J, Burgess N . 1996. G eometric determinants of the place fields of hippocampal neurons. N ature 381:425-428.

O Iton D S, Becker JT, H andelmann GE. 1979. H ippocampus, space, and memory. Brain Behav Sci 2:313-365.

0 'M ara SM , RollsET, Berthoz A, Kesner RP. 1994. N eurons responding to wholebodymotion in the primate hippocampus. J Neurosci 14:6511.

O 'Reilly RC, M cC lelland JL. 1994. H ippocampal conjunctive encoding, storage, and recall: avoiding a trade-off. H ippocampus 4:661-682.

Paulsen O, M oser El. 1998. A model of hippocampal memory encoding and retrieval: GABAergic control of synaptic plasticity. Trends N eurosci 21:273-278.

Pratt WE, M izumori SJY. 1998. Characteristics of basolateral amygdala neuronal firing on a spatial memory task involving differential reward. Behav N eurosci 11:554-570.

Rawlins JN P. 1985. Associations across time: The hippocampus as a temporary memory store. Brain Behav Sci 8:479-496.

RollsE. 1996. A theory of hippocampal function in memory. H ippocampus 6:601-620.

Samsonovich A, M cN aughton BL. 1997. Path integration and cognitive mapping in a continuous attractor neural network model. J N eurosci 17:5900-5920.

Sharp PE. 1997. Subicular cells generate similar spatial firing patterns in two geometrically and visually distinctive environments: comparison with hippocampal place cells. Behav Brain Res 85:71-92.

Skaggs WE, M cNaughton BL, Gothard KM, M arkus EF. 1993. An information-theoretic approach to deciphering the hippocampal code. In: H anson SJ, Cowan JD, Giles CL eds. Advances in neural information processing systems. San M ateo, CA: Kaufman, 10301037.
Squire LR, C ohen N J, N adel L. 1984. The medial temporal region and memory consolidation: A new hypothesis. In: Weingartner $\mathrm{H}$, Parker $\mathrm{E}$, eds. M emory consolidation. H illsdale, N J: Erlbaum, p 185-210.

Swanson LW, Sawchenko PE, Cowan W M. 1980. Evidence that the commissural, associational and septal projections of the regio inferior of the hippocampus arise fromthe same neurons. Brain Res 197:207212.

Swanson LW, Sawchenko PE, C owan W M . 1981. Evidence for collateral projections by neurons in Ammon's horn, the dentate gyrus, and the subiculum: a multiple retrograde labeling study in the rat. J N eurosci 1:548-559.

Taube JS. 1995. Head direction cells recorded in the anterior thalamic nuclei of freelymoving rats. J N eurosci 15:70-86.

Vertes RP. 1992. PH A-L analysis of projections from the supramammillary nucleus in the rat. J Comp N eurol 326:595-622.

Vertes RP, Crane AM, Colom LV, Bland BH. 1995. Ascending projections of the posterior nucleus of the hypothalamus: $\mathrm{PH} \mathrm{A-L}$ analysis in the rat. J Comp N eurol 359:90-116.

Wallenstein GV, Hasselmo ME. 1997. GABAergic modulation of hippocampal population activity: sequence learning, place field development, and the phase precession effect. J N europhysiol 78:393408.

Wallenstein GV, Eichenbaum H, H asselmo M E. 1998. The hippocampus as an associator of discontiguous events. Trends Neurosci 21:317-323.

W iener SI, Korshunov VA. 1995. Placeindependent behavioural correlates of hippocampal neurones in rats. N euroReport 7:183-188.

Wiener SI, Paul CA, Eichenbaum H. 1989. Spatial and behavioral correlates of hippocampal neuronal activity. J N eurosci 9:2737-2763.

W iener SI, Korshunov VA, G arcia R, Berthoz A. 1995. Inertial, substratal and landmark cue control of hippocampal CA1 place cell activity. Eur J N eurosci 7:2206-2219.

Wilson M A, M CNaughton BL. 1993. Dynamics of the hippocampal ensemble code for space. Science 261:1055-1058.

W ilson M A, Tonegawa S. 1997. Synaptic plasticity, place cells and spatial memory: study with second generation knockouts. Trends N eurosci 20:102-106. 